\title{
Deutsche Auslandskorrespondenten im Profil
}

\author{
Kathrin Junghanns / Thomas Hanitzsch
}

Die vorliegende Studie unternimmt den Versuch einer ersten explorativen und deskriptiven Berufsfeldanalyse, basierend auf Online-Interviews mit insgesamt 176 Auslandskorrespondenten, die für deutsche Medien berichten. Die Ergebnisse belegen, dass Auslandskorrespondenten im Durchschnitt älter und erfabrener sind als ibre Kollegen in den Heimatredaktionen. Stärker als andere Bereiche des Journalismus wird diese Domäne von Männern dominiert. Darüber hinaus neigen Auslandskorrespondenten verstärkt zu einem Rollenverständnis, das sowobl auf eine Kontextualisierung und Einordnung des Auslandsgeschehens als auch auf die kulturelle Verständigung mit der Berichtsregion setzt. Aufgrund der komplexen Anforderungen der Tätigkeit ist die Auslandskorrespondenz kein Feld für Berufseinsteiger.

Schlagwörter: Auslandskorrespondenten, Auslandsberichterstattung, Rollenselbstverständnis, Berufsfeld

In der modernen Mediengesellschaft wird unsere Wahrnehmung von Welt maßgeblich durch die Auslandsberichterstattung geprägt (vgl. Schmidt \& Wilke 1998: 169). Auslandskorrespondenten berichten aus fremden Ländern und formen damit nicht nur die Vorstellungen von anderen Kulturen, sondern bestimmen die Beziehungen zwischen den Völkern entscheidend mit (Maletzke 1966: 326). Insbesondere politische und ökonomische Prozesse laufen zunehmend medienvermittelt ab, womit den Nachrichten aus dem Ausland eine geradezu „existenzielle“ Bedeutung zukommt (Schwanebeck 2003: 30). Dies gilt vor allem für die sich unaufhaltsam globalisierende politische Arena, in der strategische Entscheidungen mit dem Blick auf internationale Konstellationen getroffen werden müssen. Insbesondere in Krisenzeiten wird der Auslandsberichterstattung ein erhebliches Beeinflussungspotenzial im Hinblick auf politische Entscheidungen bescheinigt: So legen Forschungen zum so genannten „CNN-Effekt“ nahe, dass das US-amerikanische Eingreifen in Somalia 1993 in erster Linie durch eine dramatische Medienberichterstattung herbeigeführt wurde (vgl. Robinson 2002). In ähnlicher Weise vermutet Hume (1998: 77), dass die Auslandsberichte westlicher Korrespondenten 1999 letztendlich zur NATO-Intervention im Kosovo geführt haben.

Auslandskorrespondenten besetzen daher eine Schlüsselposition in der öffentlichen Kommunikation von Auslandsgeschehen, und zuweilen liefern sie sogar die Informationsbasis, auf der Regierungen politische Entscheidungen fällen (vgl. Wu \& Hamilton 2004: 519). Angesichts der Bedeutung von Auslandsberichterstattung allgemein und Auslandskorrespondenten im Besonderen ist es in höchstem Maße erstaunlich, wie wenig wir über diejenigen wissen, die unser Bild von der Welt mit Informationen „aus erster Hand“ prägen. Und das, obwohl u. a. Maletzke bereits 1966 eine Untersuchung der „Psychologie und Soziologie“ dieser speziellen Berufsgruppe gefordert hatte. Im Unterschied zu den USA, wo bereits seit einem halben Jahrhundert empirische Forschung auf breiter Front betrieben wird, ist der Forschungsstand im deutschsprachigen Raum eher bescheiden. In den beiden großen deutschen Journalistenbefragungen (vgl. Schönbach, Stürzebecher \& Schneider 1994; Weischenberg, Löffelholz \& Scholl 1993) wurden Auslandskorrespondenten nicht als eigenständige Subgruppe ausgewiesen. Theoretisch müssten sie zumindest in der von der "Journalismus in Deutschland“-Studie ermittelten 
Gesamtzahl der Journalisten enthalten sein. Unklar ist hingegen, in welchem Umfang sie in die Stichprobe gelangten und ob hier etwa durch mangelnde Erreichbarkeit systematische Verzerrungen vorliegen.

Auslandskorrespondenten sind aufgrund ihrer berufsbedingten Dauermobilität freilich ein schwer zu greifendes Forschungsobjekt. Diese Tatsache mag erheblich dazu beigetragen haben, dass die Auslandskorrespondenten von der deutschsprachigen Journalismusforschung weitgehend ausgeblendet wurden. In diese Forschungslücke stößt die vorliegende Studie und unternimmt damit den Versuch einer ersten explorativen und deskriptiven Berufsfeldanalyse. Im Zentrum der Untersuchung stehen die spezifischen Qualifikationen und Karrierewege, das berufliche Umfeld sowie die Rollenselbstverständnisse deutscher Auslandskorrespondenten. $\mathrm{Zu}$ beantworten wird auch die Frage sein, inwieweit Auslandskorrespondenten über besondere Merkmale und Einstellungen verfügen, die es rechtfertigen, von einem besonderen „Volk “1 von Journalisten (Hannerz 2004) bzw. von einer eigenständigen „Kultur“ (Hess 2001; Pedelty 1995) zu sprechen. Aufgrund der Spezifika des Forschungsgegenstandes (Reisetätigkeit, Personalfluktuation, hohes Arbeitspensum etc.), der mangelhaften Datenlage (Informationen zu Korrespondenten sind teilweise schwer zu bekommen) sowie sehr begrenzter Ressourcen für Feldforschung wurde die Studie auf Basis eine Online-Befragung von insgesamt 176 für deutsche Medien tätige Auslandskorrespondenten realisiert.

\section{Auslandsberichterstattung als Gegenstand der Forschung}

Die Korrespondenten als Produzenten der Auslandsberichterstattung gerieten recht selten zum Gegenstand wissenschaftlicher Analysen, weshalb Marten (1987: 23) die Auslandskorrespondenten auch als „unbekannte Wesen“ der Publizistik- und Kommunikationswissenschaft bezeichnete. Zu den ersten Untersuchungen im deutschsprachigen Raum zählen die Arbeiten von Lugert (1974) über Auslandskorrespondenten in Österreich sowie von Jürgens (1974) über deutsche Korrespondenten, die für die ARD und das ZDF aus den USA berichten.

In der Bundesrepublik tätige Auslandskorrespondenten standen jeweils im Fokus der Untersuchungen von Kluge (1980), Piel (1999) und Sange (1989). Während sich Kluge mit dem Arbeitsumfeld von Berichterstattern aus Nordamerika und Europa beschäftigte, konzentrierte Piel ihre Studie auf niederländische Korrespondenten, und Sange untersuchte japanische Auslandsreporter. Korrespondenten deutscher Medien waren Gegenstand der Arbeiten von Gysin (2000), Kirschstein (1996) und Siemes (2000). Gysin gelangte in ihrer Befragung von 54 Korrespondenten Deutschschweizer Printmedien zu dem Ergebnis, dass diese in ihrer Berichterstattung die Schweizer Außenpolitik weitgehend ignorieren. Kirschstein beklagt in seiner Untersuchung von TV-Korrespondenten (NDR, WDR, RTL, VOX) eine „Liveberichterstattung im ,Feuerwehrstil'“, während Siemens aus ihrer Befragung von in Polen für deutsche Medien tätigen Auslandskorrespondenten schließt, dass angesichts der vielfältigen redaktionellen Zwänge die Rolle der Korrespondenten nicht ganz so machtvoll ist, wie der erste Blick glauben lässt.

Trotz der zum Teil stark variierenden Untersuchungsanlagen lassen sich aus dem Literaturbestand einige zentrale Tendenzen herausarbeiten²: Aufgrund der Spezifika ihrer Tätigkeit müssen Auslandskorrespondenten über besondere Qualifikationen verfügen. Häufig werden in diesem Zusammenhang Charaktereigenschaften genannt wie „Be-

1 Hannerz selbst verwendet in diesem Zusammenhang den Begriff „tribe“.

2 Vgl. dazu auch den folgenden Abschnitt. 
lastbarkeit“, „Erkundungsgeschick“, „Flexibilität“, „Neugierde“, „Sensibilität“, „Stilsicherheit" sowie ein "gutes Kontaktvermögen“ und eine „scharfe Beobachtungsgabe“ (vgl. Neudeck 1985; Tern 1982: 219; Wagner 2001: 18f.). Es scheint, als ob die längst überwunden geglaubte „Begabungsideologie“ im Journalismus (Weischenberg 1990: 11ff.) hier ihre letzte Nische gefunden hat. Nicht selten erinnern gestandene Auslandsredakteure wie Dirk Schraeder (2002: 21) vom SWR gerne an die besonderen Härten des beruflichen Alltags: „Darüber hinaus sollte der Magen alles von roher Walhaut (Grönland) über verrotteten Hering (Schweden) bis zu Hammel-Testikeln (Türkei) ertragen. Der notorische Schlafmangel in Kombination mit den erwähnten Küchen- und Klimawechseln sorgt für ein anfälliges Immunsystem."

Einigkeit besteht allenfalls darin, dass überdurchschnittliche Fremdsprachenkenntnisse unverzichtbar sind. Auch profunde Kenntnisse über das Berichterstattungsgebiet sollten dazu gehören. Die oft nicht rational zu erklärende Besetzung von Auslandskorrespondenzen führt jedoch dazu, dass sich Journalisten dieses Wissen erst mühsam vor Ort erarbeiten müssen. So berichtet Hans-Josef Dreckmann, der insgesamt 13 Jahre das ARD-Studio Nairobi geleitet hat: „Als ich das erste Mal nach Afrika kam, wusste ich herzlich wenig, zu wenig. Bis heute habe ich nicht alle Länder meines Berichtsgebietes kennengelernt." (Dreckmann in Journalistik Journal 5(1): 16)

Im Hinblick auf den Berufszugang ist festzustellen, dass Auslandskorrespondenten in der Regel eine traditionelle Journalistenausbildung durchlaufen, wobei ein abgeschlossenes Studium zunehmend an Relevanz gewinnt (vgl. Lugert 1974: 55f.; Piel 1999: 134; Siemes 2000: 114ff.; Lange 2002: 55). Um sich die nötigen Allround-Kompetenzen anzueignen, schließt sich zumeist eine mehrjährige journalistische Arbeit im Inland an, weshalb es sich bei der Tätigkeit als Auslandskorrespondent nur in seltenen Fällen um einen Einstiegsjob für Berufsanfänger handelt (vgl. Gysin 2000: 68f.; Piel 1999: 134; Siemes 2000: 115). Nicht immer entscheiden dabei objektive Kriterien über die Besetzung von Korrespondentenstellen: strategische Personalpolitik und der Zufall spielen eine nicht zu unterschätzende Rolle (vgl. Moskau 1974: 6; Wagner 2001: 17)

Mit Blick auf das Rollenselbstverständnis ist das von Jürgens (1974: 52) unter Amerikakorrespondenten beschriebene elitäre Selbstverständnis des meinungsbetonten Auslandsjournalismus im Zuge der Professionalisierung offenbar dem Bild des neutralen Vermittlers gewichen. Dies ist das Resultat einer Studie unter deutschen Polen-Korrespondenten (vgl. Siemes 2000: 116ff.). Zu ähnlichen Schlüssen gelangten Befragungen von Schweizer und niederländischen Korrespondenten (vgl. Gysin 2000: 85f.; Piel 1999: 152f.). Allerdings hat Nafroth (2002) in ihrer Untersuchung über das Japanbild deutscher Medien herausgearbeitet, dass es deutschen Auslandskorrespondenten nicht allein um die neutrale Vermittlung von Informationen geht, sondern dass diese sich auch in der Rolle des Experten sehen, der die oft komplexen Zusammenhänge erklärt und interpretiert.

Anders als im deutschsprachigen Raum ist in den USA die empirische Erforschung von im Ausland tätigen Berufskommunikatoren fest etabliert. $\mathrm{Zu}$ den „klassischen Studien" zählen die weitgehend deskriptiven Arbeiten von Anderson (1951), Kruglak (1955) und Wilhelm (1963). In ihrer Anlage prinzipiell ähnlich sind die erst kürzlich publizierten Untersuchungen von Willnat und Weaver (2003) sowie von Wu und Hamilton (2004). Während Wu und Hamilton Daten über insgesamt 354 US-Korrespondenten im Ausland erhoben haben, befragten Willnat und Weaver 152 Auslandskorrespondenten, die aus Washington und New York über die USA berichten. In methodischer Hinsicht bemerkenswert ist überdies die ethnografische Studie von Hannerz (2004). Der schwedische Anthropologe befragte mittels Tiefeninterviews insgesamt 70 Korrespondenten in Jerusalem, Johannesburg, Kapstadt und Tokio. 
Die Befunde aus älteren Untersuchungen finden sich in den neueren US-amerikanischen Studien weitgehend bestätigt: Auslandskorrespondenten sind im Durchschnitt älter und erfahrener als ihre Kollegen im Inland und verfügen über eine bessere Bildung. Darüber hinaus sind Journalistinnen hier im Vergleich zu den Heimatredaktionen unterrepräsentiert, während die Bedeutung von Korrespondenten anderer Nationalität (vorzugsweise die des Berichtslandes) über die Jahre deutlich zugenommen hat (vgl. Willnat \& Weaver 2003: 417; Wu \& Hamilton 2004: 521ff.). Mit Blick auf das Rollenverständnis haben Willnat und Weaver (2003: 415) festgestellt, dass sich Auslandskorrespondenten stärker in einer Rolle sehen, die auf die Analyse und Interpretation von komplexen Zusammenhängen abzielt.

\section{Theoretische Ansätze}

Erst in letzter Zeit häufen sich die Versuche, dem Forschungsfeld ein theoretisches Fundament zu geben (vgl. Hamilton \& Jenner 2004), auch wenn ein „übergreifendes theoretisches Modell“" nach wie vor fehlt (Siemes 2000: 25). Klassische Definitionen von Korrespondenten im Ausland sind personenzentriert und orientieren sich am Nationalstaatenkonzept. So gilt nach Marx (1982: 211) als Auslandskorrespondent, wer „außerhalb des Landes, des Staates arbeitet, in dem das Informationsorgan seinen Sitz hat". Eine solche Definition ist im Hinblick auf empirische Forschungsaktivitäten zweifellos außerordentlich praktikabel, aber theoretische Tiefe hat sie nicht.

An systemtheoretische Vorstellungen könnte eine Sicht auf Auslandsjournalismus als journalistisches Subsystem anschließen. Auslandsberichterstattung definiert sich demnach als Nachrichtenproduktion über Ereignisse, die außerhalb der nationalstaatlichen Grenzen des Territoriums stattfinden, in dem die jeweilige Redaktion hauptsächlich operiert. Hiermit wird der zentrale Referenzpunkt auf den geografischen Standort der (Haupt-)Redaktion gelegt. Allerdings mag dies aus der Perspektive des Publikums gelegentlich anders aussehen. So werden die ca. 20.000 auf Mallorca lebenden Deutschen die Berichterstattung des Spiegel über das Rauchverbot auf der Urlaubsinsel nur bedingt als Auslandsberichterstattung wahrnehmen. Darüber hinaus kann Auslandsberichterstattung auch als organisationale (Sub-)Struktur von Redaktionen beschrieben werden. Allerdings verfügen nicht alle Medienbetriebe über eine Auslandsredaktion, und Redaktionen wie die des „Weltspiegels“ (ARD) bilden eher die Ausnahme. Zudem: Selbst wenn Medien ein eigenständiges Ressort „Ausland“ unterhalten, dann wird hierunter zumeist nur das politische Weltgeschehen gefasst. Auch die übrigen Ressorts (z. B. Wirtschaft, Reise, Sport) werden von Auslandsnachrichten gespeist.

Es erscheint daher am sinnvollsten, das Konzept des Auslandskorrespondenten am Begriff der Rolle zu verankern, wobei wir - Rühl (1989) folgend - in Arbeits- und Berufsrollen unterscheiden. Arbeitsrollen stehen mit der aktuellen beruflichen Tätigkeit im Zusammenhang und werden zumeist durch organisationale Zwänge konstituiert. Ausdruck finden sie in einer vertikalen Differenzierung entlang der redaktionellen Entscheidungshierarchie, in einer horizontalen Differenzierung entlang von Ressorts und in einer für angelsächsische Redaktionen typische funktionale Trennung in Reporter-, Editor- und Kommentator-Rollen.

Die Gruppe der Auslandskorrespondenten liegt quer zu diesen Vorstellungen, sie bildet mithin eine vierte Subkategorie, die sich durch die Tätigkeit im Ausland definiert. Als „klassisches“ Rollenmodell gilt dabei das als Korrespondent entsendete Redaktionsmitglied, wobei dieses Berufbild zunehmend mit Sonderberichterstattern, Reisekorrespondenten sowie Wirtschafts- und Kulturkorrespondenten konkurriert (vgl. Schraeder 
2002; Wagner 2001). Auch in Hamilton und Jenners (2004: 313f.) Typologie von Auslandskorrespondenten kommt das klassische Modell als „traditional foreign correspondent“ vor, während Reisekorrespondenten als „parachute journalists“ und thematische Sonderkorrespondenten (z. B. Börsenberichterstattung) als „premium service foreign correspondent" bezeichnet werden. Darüber hinaus konstatieren die Autoren eine zunehmende Zahl von „foreign foreign correspondents“ (Ausländer als Korrespondenten) im Zuge von Rationalisierungsmaßnahmen in Auslandsredaktionen. ${ }^{3}$

Der Begriff der Berufsrolle reicht über die konkrete journalistische Arbeitstätigkeit hinaus und reflektiert auf die Profession als solche. Ein ganz wesentlicher Aspekt in diesem Zusammenhang ist das berufliche Rollenselbstverständnis, d. h. der „selbstgesteckte Rahmen des Handelns“ und die „Beobachtung der sich selbst zugeschriebenen Rolle“ (Weischenberg, Löffelholz \& Scholl 1994: 160). Die Erforschung des Selbstverständnisses von Journalisten hat eine lange Tradition: Während Cohen (1963: 20) noch in eine „neutrale“ und eine „Teilnehmer-Rolle“ unterschied, arbeiten Donsbach und Patterson (2003: 298ff.) mit einer zweidimensionalen Unterscheidung entlang der Pole passiv vs. aktiv sowie neutral vs. anwaltschaftlich. Auf diese Weise gelangen die Autoren zu insgesamt vier Selbstverständnis-Typen: „Passiv-neutral“ (neutrale Vermittler, Makler etc.), „Passiv-anwaltschaftlich“ (parteiisch usw.), „Aktiv-neutral“ (Spürhund, Vierte Gewalt etc.) sowie „Aktiv-anwaltschaftlich“ (Ideologen, Missionare etc.).

Im Hinblick auf Auslandskorrespondenten hat Siemes (2000: 57f.) einen Rahmen konstruiert, der es erlaubt, unterschiedliche Rollenverständnisse zu verorten. Drei Dimensionen sind hierbei von besonderer Relevanz: Die vermittelnd-erklärende Ebene umfasst all jene Aspekte, nach denen die vorrangige Aufgabe der Korrespondentenarbeit darin besteht, den Rezipienten Vorgänge und Phänomene in anderen Ländern verständlich zu machen. Diplomatisch-missionarische Ansprüche beschreiben Zielsetzungen, die auf die Beeinflussung der öffentlichen Meinung gerichtet sind, und die neutral-faktenorientierte Ebene zielt auf die wertungsfreie Weitergabe von Informationen über das Berichtsgebiet.

Oft wird im Zusammenhang mit Berufsrollen von Auslandskorrespondenten von einer eigenständigen „Kultur“ (Hess 2001; Pedelty 1995) des Elite-Journalismus (Wu \& Hamilton 2004) oder von einem „Volk“ von Gleichgesinnten (Hannerz 2004) gesprochen. Begründet wird diese Auffassung durch den Umstand, dass Korrespondenten häufig über eine besondere Ausbildung und Sachkenntnis, professionelle Netzwerke sowie über ein eigenes System der Anerkennung herausragender Leistungen verfügen (vgl. Wu \& Hamilton 2004: 519). Letztlich aber muss die Frage, ob Auslandskorrespondenten tatsächlich eine eigenständige professionelle Subkultur bilden, empirisch beantwortet werden.

Aus unserem theoretischen Verständnis und der Sichtung der einschlägigen Literatur heraus ergeben sich folgende Forschungsfragen:

1. Arbeitsrollen: Welche Charakteristika kennzeichnen deutsche Auslandskorrespondenten, hat diese Berufsgruppe ein besonderes Ausbildungsprofil vorzuweisen? Welche geografischen Schwerpunkte werden in Korrespondentennetzen gesetzt, wie ausgedehnt sind die Berichterstattungsgebiete?

3 Die verbleibenden vier von Hamilton und Jenner identifizierten Typen sind entweder nur eine Subkategorie des traditionellen Modells („foreign local correspondent“), agieren außerhalb des Journalismussystems (,amateur correspondent“) oder haben mit Auslandskorrespondenz im engeren Sinne nichts gemein („local foreign correspondent“, „,in-house foreign correspondent"). 
2. Berufsrollen: Unterscheiden sich deutsche Auslandskorrespondenten hinsichtlich ihres beruflichen Rollenverständnisses von ihren Kollegen im Inland? Kann von einer „eigenständigen Kultur“ der Auslandskorrespondenten gesprochen werden?

\section{Methode}

Da es in der vorliegenden Studie um die Erforschung von Rollenaspekten im Auslandsjournalismus unter Berücksichtigung eines breiten Spektrums an Berichterstattern ging, wurde als Erhebungsmethode die vollstandardisierte Online-Befragung gewählt. Die Methode der webbasierten Online-Befragung hat mittlerweile einen festen Platz in der empirischen Kommunikations- und Medienforschung gefunden. Diese Form der Befragung ist nicht nur extrem kosten- und zeitsparend, sie erscheint auch angemessen für eine Berufsgruppe, die berufsbedingt an den Umgang mit E-Mail und Internet gewöhnt und darin geübt ist (vgl. Wagner 2001: 14f.; Wu \& Hamilton 2004: 526). Andererseits musste mit einer geringeren Antwortbereitschaft aufgrund des fehlenden Kontakts zu einer anderen Person (z. B. Interviewer) und daraus resultierender Unverbindlichkeit gerechnet werden (vgl. Brosius \& Koschel 2001: 131f.). Zudem fällt die Problematik der Selbstselektion bei Online-Befragungen stärker ins Gewicht.

Der Fragebogen gliederte sich in fünf Themenbereiche: dem beruflichen Werdegang, der Arbeitssituation, den Arbeitsbedingungen, dem beruflichen Rollenselbstverständnis sowie den soziodemografischen Merkmalen. Um eine Vergleichbarkeit mit der „Journalismus in Deutschland"-Studie (Weischenberg, Löffelholz \& Scholl 1994) zu ermöglichen, stützten sich insbesondere die Fragen zum beruflichen Rollenverständnis auf 12 Aussagen der dort verwendeten Item-Batterie sowie auf sechs weitere Aussagen, welche die Spezifika der Auslandsberichterstattung abbilden sollten. Die beruflichen Einstellungen wurden analog zur oben zitierten Studie auf einer fünfstufigen Skala abgefragt. Nach einem Pre-Test ging die Studie vom Mai bis Juli 2004 ins Feld.

Als problematisch erwies sich erwartungsgemäß die Auswahl der zu befragenden Journalisten. Da keine aussagefähigen Daten zur Gesamtzahl deutscher Auslandskorrespondenten verfügbar sind, erfolgte die Stichprobenbildung induktiv, d. h. ohne vorherige Bestimmung der Grundgesamtheit. Ohnehin wäre die Ermittlung des Gesamtbestandes an Auslandskorrespondenten aufgrund variierender Berufsbezeichnungen, Personalfluktuation und mangelnder Auskunftsbereitschaft der Medienunternehmen ein Sisyphosgleiches Unterfangen geworden. Journalismusforscher in den USA berichten von ähnlichen Problemen (vgl. Willnat \& Weaver 2003; Wu \& Hamilton 2004). Das größte Problem bilden hierbei die freien Auslandskorrespondenten, die auf Honorarbasis arbeiten und überwiegend für mehrere Medien tätig sind. Die Medienverzeichnisse Stamm und Zimpel weisen diese Journalisten nicht gesondert als Korrespondenten aus und konnten daher für diese Zwecke nicht herangezogen werden. ${ }^{4} \mathrm{Um}$ an die Daten von deutschen Auslandskorrespondenten zu gelangen, wurden deshalb zwei Wege eingeschlagen:

1. Zunächst wurden die öffentlich-rechtlichen Rundfunkanstalten gebeten, die Kontaktdaten ihrer Auslandskorrespondenten bereitzustellen. Unter den kommerziell operierenden Medienbetrieben wurden die privaten Rundfunkanstalten mit den deutschlandweit höchsten Marktanteilen sowie die überregionalen Abonnement-Ta-

4 Nach Angaben der Zimpel-Redaktion wird diese Option jedoch schon für die nächste Aktualisierung zur Verfügung stehen. 
geszeitungen und Zeitschriften zum Zeitgeschehen mit den höchsten Reichweiten um Auskunft gebeten. ${ }^{5}$

2. Ausgehend von der Annahme, dass sich Berichterstatter in vielen Ländern akkreditieren müssen, wurden in einem weiteren Schritt die deutschen Botschaften in die Suche einbezogen. Die 103 per E-Mail erreichbaren Vertretungen wurden um Auskunft über akkreditierte Auslandskorrespondenten gebeten.

Auf diese Weise wurden 572 ständige und zeitweilige sowie feste und freie Journalisten ermittelt, die als deutsche Auslandskorrespondenten geführt werden. Nach einer ersten Kontaktaufnahme per E-Mail stellte sich heraus, dass 48 E-Mailadressen fehlerhaft bzw. eingestellt waren und 22 Journalisten bereits nicht mehr als Korrespondenten tätig waren. Die verbleibende bereinigte Stichprobe von 502 Personen resultierte in insgesamt 176 verwertbaren Interviews, was einer Rücklaufquote von 35 Prozent entspricht. ${ }^{6}$ Darunter befanden sich 154 Auslandskorrespondenten mit explizit ausgewiesener deutscher Staatsbürgerschaft. Eine Prüfung des Rücklaufs ergab keine systematischen Zusammenhänge zwischen Mediensegment und Anteil der beantworteten E-Mail-Anfragen. Zwei Drittel der befragten Korrespondenten sind für Zeitungen und Zeitschriften (66 Prozent) tätig, gefolgt von Hörfunk (32 Prozent), Fernsehen (26 Prozent), Online-Medien (10 Prozent) und Nachrichtenagenturen (6 Prozent). Immerhin ein Viertel (26 Prozent) von ihnen arbeitet cross-medial, davon die meisten zugleich für Printmedien und Hörfunksender.

\section{Befunde}

\subsection{Auslandskorrespondenz ist eine Domäne der Männer}

Die Ergebnisse der Studie nähren die Vermutung, dass es sich beim Beruf des Auslandskorrespondenten - zumindest im deutschen Journalismus - um eine Bastion männlicher Dominanz handelt. Nur knapp 22 Prozent der befragten Auslandskorrespondenten waren Journalistinnen, womit sich die vor allem von der Fernsehberichterstattung geprägte öffentliche Wahrnehmung von Auslandsjournalismus als Männerdomäne bestätigt sieht. Der Frauenanteil von etwa einem Fünftel zieht sich quer durch alle Medienbereiche hindurch und hinterlässt somit ein konsistentes Bild. Im Vergleich mit der neuesten repräsentativen Befragung von deutschen Journalisten, die einen Frauenanteil von 37 Prozent ermittelt hat (vgl. Weischenberg, Scholl \& Malik 2005: 2), fällt der Anteil von Auslandskorrespondentinnen um Einiges geringer aus. Dass dies keine spezifisch deutsche Problematik ist, haben andere Studien unter Beweis gestellt. In den USA ist der Anteil von Journalistinnen unter den Auslandskorrespondenten nach einem „dramatischen

5 Insgesamt waren dies folgende Redaktionen: ARD, ARD III (BR, SR, MDR, NDR, WDR, RBB, SWR, HR), ZDF, radio bremen, 3sat, DeutschlandRadio, Deutsche Welle, Deutschlandfunk, arte, Phoenix, RTL, RTLII, Sat.1, ProSieben, VOX, n-tv, Kabel 1, NeunLive, Antenne Bayern, Stern, Der Spiegel, Focus, SZ, FAZ, Die Welt, Handelsblatt, FR und FTD. Datengrundlage: Arbeitsgemeinschaft Fernsehforschung 2004; Allensbacher Markt- und Werbeträger-Analyse 2004.

6 Tatsächlich war der elektronische Rücklauf größer: Insgesamt haben 285 Korrespondenten auf die Umfrage reagiert, allerdings mussten 109 „Interviews“ ausgesondert werden, da - wenn überhaupt - nur wenige Einstiegsfragen beantwortet wurden. Offensichtlich haben einige Journalisten die Umfrage in Angriff genommen, sind aber sehr schnell wieder ausgestiegen, als ihnen der Zeitaufwand bewusst wurde. 
Anstieg " in den 1980er Jahren seit über einem Jahrzehnt bei etwa 26 Prozent annähernd stabil geblieben (Wu \& Hamilton 2004: 523).

Es ist zu vermuten, dass hierbei gesellschaftliche Mechanismen ganz besonders wirksam greifen, die traditionell die Zugangschancen zum sowie die Karrierewege im Berufsfeld Journalismus geschlechtsabhängig beeinflussen (vgl. den Überblick bei Lünenborg 1997). Zum einen sind es die hohen und unkalkulierbaren Arbeitszeiten im Journalismus, die es erschweren, Erwerbsarbeit und Familie miteinander zu verbinden. Journalistinnen sind hiervon in besonderem Maße betroffen, da hartnäckige gesellschaftliche Konventionen Frauen noch immer die Hauptlast der Kinderbetreuung aufbürden. Ähnliche Prozesse führen, dies ist in der gender-orientierten Journalismusforschung seit längerem bekannt, auch zu einer Benachteiligung von Journalistinnen bei der Karriereentwicklung. Zum anderen sind es oft sozial konstruierte Geschlechterimages, die Weiblichkeit mit „Weichheit“ und „Empathie“ verbinden, womit Männer für die nüchterne Berichterstattung über (politische) Sachthemen, die den Auslandsjournalismus dominiert, eher geeignet erscheinen (vgl. Keuneke, Kriener \& Meckel 1997: 37).

Lavie und Lehman-Wilzig (2003: 21) vermuten daher, dass unter diesen Bedingungen viele Journalistinnen bereits an einem frühen Punkt ihrer Karriere dem Berufsfeld des Nachrichtenjournalismus den Rücken kehren. Es ist anzunehmen, dass diese Problematik angesichts der spezifischen beruflichen Herausforderungen unter Auslandskorrespondenten von ganz besonderer Relevanz ist. Ob der geringe Anteil von deutschen Korrespondentinnen ein Resultat dieser Vermeidungsstrategie oder mehr oder weniger bewusster beruflicher Diskriminierung ist, kann die vorliegende Studie nicht beantworten.

Auslandskorrespondentinnen sind durchschnittlich fast acht Jahre jünger als ihre männlichen Kollegen, wobei die jüngste Berichterstatterin der Stichprobe 23 Jahre und der älteste Korrespondent 67 Jahre alt waren. Dieser Befund ist für die gender-orientierte Journalismusforschung keine Überraschung, da sich auch in allgemeinen Journalistenbefragungen gezeigt hat, dass Journalistinnen in der Regel jünger sind (vgl. u. a. Chambers, Steiner \& Fleming 2004: 102). Die Altersdifferenz zwischen Journalistinnen und Journalisten weist einmal mehr auf die Problematik der Unvereinbarkeit von Beruf und Familienarbeit hin, die viele Auslandskorrespondentinnen dazu veranlassen mag, entweder ganz aus dem Beruf auszusteigen oder auf eine besser kalkulierbare Tätigkeit im Journalismus auszuweichen.

\subsection{Auslandskorrespondenten sind erfabrene Journalisten}

Deutsche Auslandskorrespondenten können eine überdurchschnittliche Berufserfahrung von 20 Jahren vorweisen. Durchschnittlich zehn Jahre ist ein Auslandskorrespondent journalistisch tätig, bevor er sich für eine Korrespondentenstelle entscheidet. Journalistinnen haben im Mittel fast drei Jahre weniger Berufserfahrung vorzuweisen als ihre männlichen Kollegen, wenn sie auf eine Korrespondenz wechseln. Angesichts dieser Befunde lassen sich Auslandskorrespondenten durchaus als besonders erfahrene Journalisten beschreiben, denen erst im fortgeschrittenen Stadium ihrer Karriere eine solche Tätigkeit zugetraut wird oder die nach einer längeren und möglicherweise verdienstvollen redaktionellen Arbeit mit einem lukrativen Posten als Auslandskorrespondent „belohnt" werden. Immerhin sind 53 Prozent der befragten Korrespondenten vor ihrer Auslandstätigkeit bereits mindestens zehn Jahre als Journalist tätig gewesen. Allerdings: Jeder zehnte befragte Auslandskorrespondent ist direkt in diese Tätigkeit eingestiegen, 
d. h. weitgehend ohne praktische Berufserfahrung. Hierbei handelt es sich vor allem um freie und nebenberuflich tätige Korrespondenten.

Die von uns befragten Auslandskorrespondenten waren im Durchschnitt knapp 44 Jahre alt, womit sie sich auch in dieser Hinsicht vom Berufsgruppen-Querschnitt unterscheiden (41 Jahre, vgl. Weischenberg, Scholl \& Malik 2005: 2). US-amerikanische Studien kommen zu ähnlichen Ergebnissen und betonen den elitären Charakter dieser Berufsgruppe (Wu und Hamilton 2004: 521). Doch schlagen sich diese Besonderheiten auch in einem spezifischen Ausbildungsprofil nieder? Tabelle 1 lässt erkennen, dass ein journalistisches Praktikum bzw. eine Hospitanz sowie das Volontariat die Hauptzugangswege in den Beruf markieren. Nur knapp jeder zehnte befragte Auslandskorrespondent hat ein Studium der Journalistik absolviert, aber immerhin fast jeder Fünfte eines der Publizistik, Kommunikations- oder Medienwissenschaft. Keine journalismusrelevante Ausbildung hat etwa ein Fünftel der Befragten vorzuweisen.

Damit unterscheiden sich Auslandskorrespondenten nicht gravierend von ihren Kollegen im Inland. Es lässt sich allenfalls anmerken, dass die Durchdringung des Berufsfeldes mit Journalisten, die ein Praktikum und/oder ein Volontariat absolviert haben, im Vergleich zum gesamten Berufsstand etwas geringer ist. Insgesamt können 74 Prozent der Auslandskorrespondenten einen Studienabschluss vorweisen, wobei auch dieser Wert nur leicht über dem Durchschnitt liegt (69 Prozent, vgl. Weischenberg, Scholl \& Malik 2005: 2). Die These vom besonderen Ausbildungsprofil lässt sich demnach für deutsche Auslandskorrespondenten nicht halten.

\section{Tabelle 1: Journalistische Ausbildungswege (N=176, Mebrfachnennungen möglich)}

\begin{tabular}{lcc}
\hline & $\begin{array}{c}\text { Auslands- } \\
\text { korrespondenten }\end{array}$ & $\begin{array}{c}\text { Journalisten in } \\
\text { Deutschland* }\end{array}$ \\
\hline Hospitanz / Praktikum & $46,6 \%$ & $68,7 \%$ \\
Volontariat & $42,6 \%$ & $62,4 \%$ \\
Studium der Kommunikations- und Medienwissen- & $18,8 \%$ & $17,1 \%$ \\
schaft, Publizistik & & \\
Journalistenschule & $16,5 \%$ & $13,7 \%$ \\
Studium der Journalistik & $10,8 \%$ & $13,5 \%$ \\
\hline keine journalismusrelevante Ausbildung & $18,8 \%$ & $\mathrm{k}$. A. \\
\hline
\end{tabular}

* Quelle: Weischenberg, Scholl \& Malik (2005: 2)

Unter den Auslandskorrespondenten, die ein Fachstudium absolviert haben, rangieren die historisch-philologischen Fächer mit 42 Prozent vor den politikwissenschaftlichen (37 Prozent) und den wirtschaftswissenschaftlichen Studienrichtungen (28 Prozent). Vielfach wurde auch eine Kombination aus diesen (und anderen) Fächern studiert. Unter den historisch-philologischen Fächern spielen insbesondere die Geschichtswissenschaft (25 Prozent) sowie kulturspezifische Philologien (Afrikanistik, Japanologie, Romanistik, Sinologie etc.) eine herausragende Rolle. Insgesamt bleibt festzuhalten, dass ein regionalspezifisches Fachstudium für jene Auslandskorrespondenten, die ein kulturell recht kohärentes Berichtsgebiet bearbeiten, von großem Vorteil ist. Für diejenigen Auslandkorrespondenten, die sich mit einem ausgedehnten und zum Teil sehr heterogenen Berichtsgebiet (z. B. „Afrika“ oder „Ost-, Südost- und Südasien“) konfrontiert sehen, ist eine solche berufliche Vorbereitung jedoch kaum möglich. 


\subsection{Standortwabl bestätigt Eurozentrismus der Nachrichtengeografie}

Die wenigsten Medienbetriebe in Deutschland leisten sich ein ähnlich ausgedehntes Korrespondentennetz wie die ARD (25 Standorte) und der Spiegel (22). Einzig die Deutsche Presseagentur (dpa) unterhält ein eng gewebtes Korrespondentennetz, das auf immerhin 120 Standorten in 105 Ländern aufbaut - davon allein sieben in den USA. In anderen Medienbetrieben erreichen die Berichterstattungsgebiete deutscher Auslandskorrespondenten daher zuweilen gigantische geografische Ausmaße. Dabei werden nicht selten politisch und kulturell heterogene Einheiten gebündelt, wie etwa das arabisch-islamisch geprägte Nordafrika mit dem subsaharischen Afrika. Durchschnittlich müssen die Korrespondenten, die an unserer Studie teilgenommen haben, das Geschehen in etwas mehr als sieben Ländern im Auge behalten.

Besonders ausgedehnte Berichterstattungsgebiete bearbeiten dabei Afrika-Korrespondenten, die oft von Nairobi und Johannesburg aus operieren, sowie ihre Kollegen, die für Lateinamerika (aus Rio de Janeiro) oder Australien und den pazifischen Raum (aus Melbourne) zuständig sind. Immerhin neun Prozent der befragten Auslandskorrespondenten müssen über mehr als 20 Länder berichten, 16 Prozent über elf bis 20 Länder. Üblicherweise berichten Korrespondenten über ein Berichterstattungsgebiet, das zwischen zwei und fünf Länder (38 Prozent) bzw. zwischen sechs und zehn Länder (19 Prozent) umfasst. Nur 17 Prozent derer, die an der Studie partizipiert haben, berichten über ein einziges Land. Hierunter befinden sich vor allem EU-Berichterstatter und USA-Korrespondenten.

Eine Übersicht über die Haupt-Berichterstattungsgebiete bestätigt das Bild, das Forschungen zur deutschen Nachrichtengeografie gezeichnet haben (vgl. u.a. Schmidt \& Wilke 1998: 178ff.). Der Standort Europa dominiert erwartungsgemäß unter den Auslandsbüros (vgl. Tabelle 2). Dahinter folgen der von Konflikten überschattete Nahe und Mittlere Osten sowie Asien, das vermutlich wegen seiner zunehmenden Wirtschaftskraft stärker Bedeutung erlangt. Damit schlägt sich die Europa-Dominanz zumindest nominell auch in der Verteilung der Korrespondentenbüros nieder. Allerdings wird die EU-Berichterstattung im Zuge des europäischen Integrationsprozesses zunehmend eine

Tabelle 2: Korrespondenten nach Haupt-Berichterstattungsgebieten ( $N=176)$

\begin{tabular}{lc}
\hline Haupt-Berichterstattungsgebiet & Anteil Auslandskorrespondenten \\
\hline Europa & $44,9 \%$ \\
Naher und Mittlerer Osten & $18,2 \%$ \\
Asien* & $11,4 \%$ \\
Nordamerika & $7,4 \%$ \\
Afrika & $6,3 \%$ \\
Lateinamerika & $5,7 \%$ \\
GUS-Staaten & $4,0 \%$ \\
Australien und Pazifik & $2,3 \%$ \\
\hline * Dieser Raum schließt die Länder Asiens ohne GUS und den Nahen/Mittleren Osten ein. Im Wesentlichen \\
sind dies Ost-, Süd- und Südostasien sowie Teile Zentralasiens.
\end{tabular}

7 Bei EU-Korrespondenten, die überwiegend von Brüssel aus berichten, wurde die Zahl der Länder auf 1 gesetzt. „Palästina“ wurde als Land gezählt, auch wenn es sich nicht um einen unabhängigen Staat handelt. 
Sonderstellung einnehmen, die zwischen Inlands- und Auslandsberichterstattung anzusiedeln ist.

Die Grenzen zwischen den Haupt-Berichterstattungsgebieten sind dabei nicht selten durchlässig. Manche Korrespondenten müssen Nordamerika mit dem US-amerikanisch stark beeinflussten Mittelamerika kombinieren, andere berichten von Singapur aus über ganz Südostasien, Australien und Neuseeland und müssen oft beträchtliche geografische Distanzen überwinden. In einigen Fällen bedienen Journalisten den Nahen und Mittleren Osten plus das arabisch-islamisch geprägte Nordafrika, wohingegen vor allem auf Kriegsschauplätze spezialisierte Korrespondenten neben dem Nahen und Mittleren Osten auch über Afghanistan und Pakistan berichten - sehr wahrscheinlich, weil beide Länder im Kampf gegen den islamistischen Terror eine wichtige Rolle spielen. Typische Länderkombinationen sind Großbritannien und Irland sowie die USA mit Kanada. Häufig bilden die skandinavischen Länder ein gemeinsames Berichterstattungsgebiet, ebenso Osteuropa und die Balkanstaaten, wobei die Korrespondenten nicht selten in Wien stationiert sind.

In vielen Fällen müssen Auslandskorrespondenten über ein politisch, wirtschaftlich, kulturell und sozial heterogenes Gebiet berichten, was eine hinreichende Vorbereitung im Hinblick auf Länder- und Sprachkompetenz von vornherein unmöglich macht. Dies könnte möglicherweise über Erfahrung kompensiert werden, die sich Korrespondenten über eine langjährige Tätigkeit im jeweiligen Berichtsgebiet aneignen können. Im Durchschnitt berichteten die befragten Journalisten bereits seit reichlich sieben Jahren aus ihrem derzeitigen Berichtsgebiet. Immerhin 58 Prozent können eine Erfahrung von fünf und mehr Jahren vorweisen, und 25 Prozent sind bereits seit mindestens zehn Jahren in der Region tätig.

Die von uns befragten Auslandskorrespondenten waren überwiegend hauptberuflich tätig (93 Prozent) und bei mindestens einem Medienunternehmen fest angestellt (60 Prozent). Etwa 24 Prozent der Korrespondenten arbeiten als feste Freie und 16 Prozent als freie Journalisten. Gelegentlich sind festangestellte Auslandskorrespondenten noch frei für andere Medien tätig, dies war in reichlich zwei Prozent der befragten Journalisten der Fall. Nach den vorliegenden Befunden zu urteilen, ist die Zahl der Ausländer unter den Korrespondenten („foreign foreign correspondents“, Hamilton \& Jenners 2004) relativ gering. Am höchsten ist er allerdings unter den freien Journalisten, wovon wahrscheinlich insbesondere auf Honorarbasis tätige freiberufliche Korrespondenten („Stringer“) erfasst werden, die als Ortsansässige die Korrespondentenbüros mit Informationen und Texten versorgen. Leider liegen für die Vergangenheit keine Zahlen vor, so dass in diesem Beitrag keine Aussagen über eventuelle Veränderungen in den Anstellungsverhältnissen getroffen werden können.

\subsection{Verständigungsorientierter neutraler Informationsvermittler dominiert}

Die von Nafroth (2002) aufgestellte These, dass es die für deutsche Medien berichtenden Auslandskorrespondenten für extrem wichtig halten, komplexe Sachverhalte zu erklären sowie Zusammenhänge und Bezüge herzustellen, findet sich in der vorliegenden Studie bestätigt (vgl. Tabelle 3). ${ }^{8}$ Allerdings rangiert das Verständnis der neutralen und präzisen Information auf dem vordersten Platz. Etwas weiter abgeschlagen befinden sich die Intentionen, dem Publikum Hilfe bei dessen Meinungsbildung anzubieten, schnell zu informieren, Nachrichten zu liefern, die für ein möglichst breites Publikum interessant

8 Skala: $1=$ „Aussage trifft auf mich vollkommen zu“ $\ldots 5=$ „Aussage trifft nicht $z u “$. 
sind, sowie (politische) Missstände aufzudecken. Wichtig scheint deutschen Auslandskorrespondenten auch ein Verständnis zu sein, wonach Auslandsjournalismus eine aktive Rolle bei der Prägung der Wahrnehmung des Auslands spielen soll. Demnach wollen Auslandskorrespondenten Verständnis und Problembewusstsein für das Berichtsland fördern sowie Interesse für das Berichtsgebiet wecken. Weitere Kommunikationsziele, die ein solches, eher interventionistisches Rollenverständnis anzeigen, rangieren eher im Mittelfeld: „Vorurteile und Berührungsängste gegenüber dem Berichtsland abbauen“, „als Mittler zwischen dem Berichtsgebiet und Deutschland fungieren“ sowie „einen Dialog herstellen und Verständigung ermöglichen“. Allerdings zeigt die hohe Standardabweichung für das Mittler-Verständnis $(s=1,23)$, dass dieser Punkt unter den befragten Journalisten am umstrittensten war. Insgesamt für weniger wichtig erachten Auslandskorrespondenten ein meinungsbetontes und anwaltschaftliches Rollenverständnis sowie die Versorgung des Publikums mit Unterhaltung und Entspannung.

\section{Tabelle 3: Rollenverständnis von Auslandskorrespondenten ( $N=154$, Mittelwerte)}

\begin{tabular}{|c|c|c|c|}
\hline Kommunikationsziele & Frauen & Männer & gesamt \\
\hline das Publikum möglichst neutral und präzise informieren & 1,62 & 1,56 & 1,57 \\
\hline komplexe Sachverhalte erklären & 1,62 & 1,70 & 1,68 \\
\hline Zusammenhänge und Bezüge herstellen & 1,51 & 1,74 & 1,69 \\
\hline $\begin{array}{l}\text { Verständnis und Problembewusstsein für das Berichtsland } \\
\text { fördern }\end{array}$ & 1,58 & 1,83 & 1,78 \\
\hline Interesse für das Berichtsgebiet wecken & 1,62 & 2,01 & 1,93 \\
\hline dem Publikum Hilfe bei der Meinungsbildung anbieten & 2,03 & 1,95 & 1,97 \\
\hline das Publikum möglichst schnell informieren & 2,21 & 2,07 & 2,10 \\
\hline $\begin{array}{l}\text { Nachrichten liefern, die für ein möglichst breites Publikum } \\
\text { interessant sind }\end{array}$ & 2,16 & 2,22 & 2,21 \\
\hline $\begin{array}{l}\text { Vorurteile und Berührungsängste gegenüber dem Berichtsland } \\
\text { abbauen }\end{array}$ & 1,82 & 2,45 & 2,31 \\
\hline $\begin{array}{l}\text { als Mittler zwischen dem Berichtsgebiet und Deutschland } \\
\text { fungieren }\end{array}$ & 2,39 & 2,51 & 2,49 \\
\hline (politische) Missstände aufdecken & 2,21 & 2,73 & 2,62 \\
\hline einen Dialog herstellen und Verständigung ermöglichen & 2,38 & 2,84 & 2,75 \\
\hline „normalen“ Leuten die Chance geben, ihre Meinung zu sagen & 2,86 & 3,23 & 3,15 \\
\hline mich für Benachteiligte in der Bevölkerung einsetzen & 2,97 & 3,33 & 3,25 \\
\hline dem Publikum Unterhaltung und Entspannung bieten & 3,53 & 3,36 & 3,39 \\
\hline eine persönliche Sicht auf das Geschehen mitteilen & 3,18 & 3,46 & 3,40 \\
\hline Themen auf die (politische) Agenda setzen & 3,68 & 3,76 & 3,74 \\
\hline $\begin{array}{l}\text { einen Gegenpart zu den Bereichen Politik und Wirtschaft zu } \\
\text { bilden }\end{array}$ & 3,49 & 3,89 & 3,80 \\
\hline
\end{tabular}

Aus den vorliegenden Befunden lässt sich folgern, dass die für deutsche Medien berichtenden Auslandskorrespondenten wie ihre Kollegen im Inland hauptsächlich dem Verständnis des neutralen und objektiven Informationsvermittlers zuneigen, dabei jedoch durchaus verständigungsorientiert wirken wollen. Ihre Rolle als unmittelbarer Augenzeuge des Geschehens vor Ort schließt im Verständnis vieler Berichterstatter den Auftrag mit ein, beim Publikum Interesse für das Berichtsgebiet und insbesondere für 
dessen spezifische Probleme zu wecken. Allerdings neigen Auslandskorrespondenten dabei weniger zu der Auffassung, dass es wichtig sei, auf eine Veränderung politischer Zustände hinzuwirken, sei es durch gezielte Themensetzungen oder eigene Meinungsäußerungen.

Zudem lassen sich einige Segregationslinien ausmachen, die zwischen die befragten Auslandskorrespondenten quer hindurch gehen. Zum einen gibt es Hinweise darauf, dass Journalisten, die überwiegend aus Entwicklungs- und Schwellenländern berichten, stärker ein verständigungsorientiertes und anwaltschaftliches Rollenverständnis betonen. Sie wollen mehr als andere das Verständnis und Problembewusstsein für die Berichtsregion fördern $(\bar{x}=1,65$ vs. $\bar{x}=1,88)$, Vorurteile und Berührungsängste abbauen $(\bar{x}=2,07$ vs. $\bar{x}=2,51)$, sich für Benachteiligte in der Bevölkerung einsetzen $(\bar{x}=3,00$ vs. $\bar{x}=3,47)$, einen Gegenpart zu Politik und Wirtschaft bilden $(=3,61$ vs. $\bar{x}=3,97)$ sowie „normalen“ Leuten die Chance zur Meinungsartikulation geben $(\bar{x}=3,01$ vs. $\bar{x}=3,27)$. Darüber hinaus neigen insbesondere weibliche Auslandskorrespondenten stärker zu einem Selbstverständnis der Verständigung, Kontextualisierung, kritischen Kontrolle und Meinungsäußerung. Korrespondentinnen setzen sich stärker als ihre männlichen Kollegen dafür ein, Vorurteile und Berührungsängste abzubauen, Interesse für das Berichtsgebiet zu wecken, einen Dialog herzustellen sowie Verständnis und ein Problembewusstsein für das Berichtsland zu fördern. Darüber tendieren sie stärker zu einem anwaltschaftlichen, politischen und meinungsbetonten Rollenverständnis (vgl. Tabelle 3). Dies steht im Widerspruch zu Befunden aus allgemeinen Journalistenbefragungen, wonach das Geschlecht im Hinblick auf das berufliche Rollenverständnis von eher untergeordneter Bedeutung ist (vgl. Scholl \& Weischenberg 1998: 251ff.). Welche Gründe für das unterschiedliche Selbstverständnis verantwortlich sind, sollte durch weitere Forschungen aufgeklärt werden.

Um der Frage nach möglichen Unterschieden zwischen deutschen Auslandskorrespondenten und ihren Kollegen im Inland nachzugehen, erschien es sinnvoll, die Stichprobe auf jene 154 Journalisten zu reduzieren, die sich als deutsche Staatsbürger ausgewiesen haben. Andernfalls wäre die Gefahr zu groß, dass die betrachteten Differenzen von kulturellen Faktoren überlagert werden, die sich aus der Zugehörigkeit zu verschiedenen nationalen Kontexten erklären. Der Vergleich mit Befunden aus der ersten Studie „Journalismus in Deutschland“ (die neueren Befunde zum Rollenverständnis sind noch nicht publiziert) mag hier trotz des großen zeitlichen Abstandes beider Datenerhebungen (12 Jahre) nicht ganz so prekär erscheinen, da es Anzeichen dafür gibt, dass sich journalistische Rollenverständnisse selbst über einen längeren Zeitraum hinweg nicht dramatisch verändern (Weaver et al. 2006).

Eine Gegenüberstellung der Daten deutet auf überraschend starke Unterschiede zwischen deutschen Auslandskorrespondenten und ihren Kollegen im Inland hin (vgl. Tabelle 4). Auslandskorrespondenten halten es für wichtiger, ihr Publikum möglichst neutral und präzise zu informieren sowie komplexe Sachverhalte zu erklären. Dahinter steht das konventionelle Konzept des neutralen und objektiven Berichterstatters, der ausgerüstet mit fundiertem Hintergrundwissen - das Geschehen im Ausland erklärt und in komplexe Zusammenhänge einordnet. Die abschließende Bewertung der Ereignisse wird dabei zumeist der Heimatredaktion überlassen. Deutsche Auslandskorrespondenten möchten deutlich weniger als ihre Kollegen im Inland ihren persönlichen Blick auf das Geschehen in die Berichterstattung einfließen lassen. Darüber hinaus erachten sie es für besonders wichtig, mit ihren journalistischen Beiträgen ein möglichst breites $\mathrm{Pu}-$ blikum anzusprechen. Dies ist möglicherweise im Zusammenhang mit dem sehr stark verständigungsorientierten Rollenverständnis von Auslandskorrespondenten zu sehen. 
Weniger wichtig als ihren Kollegen in der Heimatredaktion scheint Auslandskorrespondenten jedoch ein anwaltschaftliches Selbstverständnis zu sein, das sich auf Benachteiligte in der Bevölkerung sowie die Artikulationschancen von "normalen“ Leuten richtet. Auch geht es ihnen weniger darum, bestimmte - möglicherweise politische - Themen auf die Agenda zu setzen sowie einen Gegenpart zu den Bereichen Politik und Wirtschaft zu bilden. Der von dem renommierten ehemaligen BBC-Korrespondenten Martin Bell (1997) propagierte „journalism of attachment“, der versucht, durch aktive Themensetzung bestimmte politische Entscheidungen herbeizuführen (z. B. 1999 den NATO-Einsatz im Kosovo), scheint unter Auslandskorrespondenten insgesamt nur eine marginale Rolle zu spielen. Im Ausland tätige Berichterstatter legen zudem ungleich weniger Wert darauf, ihrem Publikum Unterhaltung und Entspannung zu bieten, was sich leicht mit der Dominanz von politischen Auslandskorrespondenten erklären lässt.

\section{Tabelle 4: Rollenverständnis von Auslandskorrespondenten mit deutschem Pass im Vergleich (N=154, Mittelwerte)}

\begin{tabular}{|c|c|c|}
\hline Kommunikationsziele & $\begin{array}{l}\text { Auslands- } \\
\text { korrespondenten* }\end{array}$ & $\begin{array}{l}\text { Journalisten in } \\
\text { Deutschland } * *\end{array}$ \\
\hline $\begin{array}{l}\text { das Publikum möglichst neutral und präzise } \\
\text { informieren }\end{array}$ & 1,57 & 1,97 \\
\hline komplexe Sachverhalte erklären & 1,66 & 1,97 \\
\hline $\begin{array}{l}\text { Nachrichten liefern, die für ein möglichst breites } \\
\text { Publikum interessant sind }\end{array}$ & 2,19 & 2,60 \\
\hline das Publikum möglichst schnell informieren & 2,04 & 2,01 \\
\hline $\begin{array}{l}\text { „normalen“ Leuten die Chance geben, ihre } \\
\text { Meinung zu sagen }\end{array}$ & 3,24 & 2,88 \\
\hline $\begin{array}{l}\text { mich für Benachteiligte in der Bevölkerung } \\
\text { einsetzen }\end{array}$ & 3,34 & 2,74 \\
\hline $\begin{array}{l}\text { meinen persönlichen Blick auf die Geschehnisse } \\
\text { mitteilen }\end{array}$ & 3,41 & 3,20 \\
\hline $\begin{array}{l}\text { dem Publikum Unterhaltung und Entspannung } \\
\text { bieten }\end{array}$ & 3,42 & 2,72 \\
\hline Themen auf die (politische) Agenda setzen & 3,75 & 3,55 \\
\hline $\begin{array}{l}\text { einen Gegenpart zu den Bereichen Politik und } \\
\text { Wirtschaft zu bilden }\end{array}$ & 3,83 & $3,05 / 3,36 * * *$ \\
\hline $\begin{array}{ll}* & \text { Nur mit deutscher Staatsbürgerschaft } \\
\because * & \text { Quelle: Weischenberg, Löffelholz \& Scholl (1998: } 2 \\
* * & \text { Gegenpart zu Politik } 3 \text {. }\end{array}$ & & \\
\hline
\end{tabular}

\section{Resümee und Ausblick}

Die vorliegenden Befunde sind ein kleiner Schritt zu einem besseren Verständnis von Auslandskorrespondenten, die für deutsche Medien tätig sind. Auch wenn die Daten trotz des Stichprobenumfanges ( $\mathrm{N}=176$ ) keinen Anspruch auf Repräsentativität erheben können: Auf den ersten Blick scheint sich tatsächlich zu bestätigen, dass jene, die uns vor Ort mit Informationen über das Ausland versorgen, im Gesamtkonzert der deutschen Journalisten eine besondere Subgruppe bilden. Auslandskorrespondenten sind im Durchschnitt älter und erfahrener, stärker als andere Bereiche des Journalismus wird 
diese Domäne von Männern dominiert. Aufgrund der komplexen Anforderungen der Tätigkeit ist Auslandskorrespondenz auch kein Feld für Berufseinsteiger.

Darüber hinaus setzen Auslandskorrespondenten stärker auf ein Rollenverständnis, das auf die Kontextualisierung und Einordnung des Auslandsgeschehens setzt sowie die kulturelle Verständigung mit der Berichtsregion im Auge behält. Damit konnte die vorliegende Untersuchung eine bislang von der Journalismusforschung weitgehend ignorierte Dimension der beruflichen Rollenselbstwahrnehmung herausarbeiten: eine Verständnis- und Dialogorientierung. Ein solches, eher interventionistisches Rollenverständnis weist den Auslandskorrespondenten eine aktive Rolle bei der Prägung der Auslandswahrnehmung sowie bei der Herstellung von Problembewusstsein und dem Abbau von Vorurteilen zu. Ob dies genügt, um Auslandskorrespondenten als eigenständige „Kultur“ (Hess 2001; Pedelty 1995) oder als „Volk“ von Gleichgesinnten (Hannerz 2004) zu kennzeichnen, ist allerdings angesichts der von uns ermittelten Unterschiede zwischen den befragten Korrespondenten - insbesondere die Differenzen zwischen Journalistinnen und ihren männlichen Kollegen - mehr als zweifelhaft. Besondere Ausbildungswege sind im deutschen Auslandsjournalismus zudem ebenfalls nicht erkennbar, was sicherlich dem Umstand geschuldet ist, dass viele Korrespondenten über ein geografisch ausgedehntes und kulturell heterogenes Gebiet berichten müssen, womit eine spezifische Vorbereitung (Fachstudium, Sprachkenntnisse etc.) oft kaum möglich ist. Wenn journalistische Kulturen unausgesprochene Konventionen im Hinblick auf die berufliche Selbstwahrnehmung anleiten, so gilt dies - zumindest zum gegenwärtigen Zeitpunkt - nicht für Auslandskorrespondenten, dazu sind die einzelnen Rollenverständnisse der befragten Journalisten zu unterschiedlich ausgefallen.

Künftige Forschungen zu Auslandskorrespondenten sollten die Heimatredaktionen noch stärker in die Betrachtung mit einbeziehen, um den Prozess der Aussagenentstehung im Auslandsjournalismus vollständiger abbilden zu können. Im Vordergrund sollte hierbei die Problematik der redaktionellen Organisation stehen: Wie funktioniert die Abstimmung mit der Heimatredaktion? Wie sehen die Entscheidungsabläufe aus? Welche Rolle spielen Auslandskorrespondenten bei der Auswahl von Themen und Berichterstattungsperspektiven? Wie hoch ist der Grad der redaktionellen Unabhängigkeit von Auslandsberichterstattern? Was geschieht, wenn Korrespondenten in Konflikt mit ihrer Redaktion geraten?

Um die Forschung über Auslandskorrespondenten besser an die - weitgehend inhaltsanalytisch gespeiste - Diskussion über Nachrichtenflüsse und Nachrichtenwerte anzuschließen, wäre zu fragen, welchen Berichterstattungsthemen Korrespondenten das höchste Gewicht geben. Damit wäre u. a. zweifelsfrei zu belegen, inwieweit die Dominanz von Konflikten, Katastrophen und Sensationen tatsächlich auf das Wirken von Auslandskorrespondenten als Individuen zurückgeht oder ob - was neuere Forschungen nahe legen (vgl. u. a. Richter 1999; Wolfsfeld 1997) - es sich nicht eher um ein strukturelles Problem des Journalismus handelt. Hierfür müssten allerdings inhaltsanalytische Daten mit Befragungsdaten verknüpft werden, denn nur so lässt sich das in der Journalismusforschung bislang ungelöste Problem der Handlungsrelevanz von beruflichen Einstellungen lösen. Darüber hinaus hält die qualitative Forschung eine Vielzahl von Ansätzen und Methoden bereit, um eine „dichte Beschreibung“ des Arbeitsalltages und der Karrierewege von Auslandskorrespondenten zu liefern. Hier sind insbesondere ethnografische Redaktionsstudien gefragt, wie sie u. a. Hannerz (2004) ausgearbeitet hat. Damit wären nicht zuletzt auch Rückschlüsse auf die besondere Situation von freiberuflichen Auslandskorrespondenten möglich, die das Bild des Auslandsjournalismus immer stärker prägen. 


\section{Literatur}

Anderson, Russell F. (1951): News from Nowhere. In: The Saturday Review of Literature, 17.11.1951.

Bell, Martin (1997): TV News: How Far Should We Go? In: British Journalism Review 8 (1): 716.

Brosius, Hans-Bernd \& Friederike Koschel (2001): Methoden der empirischen Kommunikationsforschung. Eine Einführung. 2., überarb. Aufl. Wiesbaden: Westdeutscher Verlag.

Chambers, Deborah, Linda Steiner \& Carole Fleming (2004): Women and Journalism. London \& New York: Routledge.

Cohen, Bernhard Cecil (1963): The Press and Foreign Policy. Princeton, NJ: Princeton University Press.

Donsbach, Wolfgang \& Thomas Patterson (2003): Journalisten in der politischen Kommunikation: Professionelle Orientierungen von Nachrichtenredakteuren im internationalen Vergleich. In: Frank Esser \& Barbara Pfetsch (Hrsg.): Politische Kommunikation im internationalen Vergleich. Grundlagen, Anwendungen, Perspektiven. Wiesbaden: Westdeutscher Verlag. 281-304.

Gysin, Nicole (2000): Der direkte Draht zur Welt? Eine Untersuchung über Auslandskorrespondentinnen und -korrespondenten Deutschschweizer Printmedien. Berner Texte zur Medienwissenschaft 5. Bern: Institut für Medienwissenschaft, Universität Bern.

Hamilton, John Maxwell \& Eric Jenner (2004): Redefining Foreign Correspondence. In: Journalism 5 (3): 301-321.

Hannerz, Ulf (2004): Foreign News: Exploring the World of Foreign Correspondents. Chicago, IL: University of Chicago Press.

Hess, Stephen (2001): The Culture of Foreign Correspondents. In: Jeremy Tunstall (ed.): Media Occupations and Professions: A Reader. Oxford: Oxford University Press. 162-169.

Hume, Mick (1998): Nazifying the Serbs, from Bosnia to Kosovo. In: Philip Hammond \& Edward S. Herman (eds.): Degraded Capability: The Media and the Kosovo Crisis. London \& Sterling, VA: Pluto Press. 70-78.

Jürgens, Georg (1974): Politische Auslandsberichterstattung im deutschen Fernsehen am Beispiel der Korrespondentenberichterstattung über die USA. Berlin: Leitstelle politische Dokumentation.

Keuneke, Susanne, Markus Kriener \& Miriam Meckel (1997): Von Gleichem und Ungleichem. Frauen im Journalismus. In: Rundfunk und Fernsehen, 45 (1), 30-45.

Kirschstein, Frank (1996): Liveberichterstattung im ,Feuerwehrstil’. Auswirkungen neuer Technologien auf die Auslandsberichterstattung. In: Miriam Meckel \& Markus Kriener (Hrsg.): Internationale Kommunikation. Eine Einführung. Opladen: Westdeutscher Verlag. 229-240.

Kluge, Herbert (1980): Die Bundesrepublik Deutschland als Objekt der Auslandsberichterstattung. Die Arbeit ausländischer Korrespondenten in der Bundesrepublik. Dissertation, Universität Münster.

Kruglak, Theodore E. (1955): The Foreign Correspondents: A Study of the Men and Women Reporting for the American Information Media in Western Europe. Geneva: E. Droz.

Lavie, Aliza \& Sam Lehman-Wilzig (2003): Whose News? Does Gender Determine the Editorial Product? In: European Journal of Communication, 18 (1), 5-29.

Lange, Silvia (2002): Deutschsprachige Auslandskorrespondenten in Lateinamerika. Magisterarbeit, Freie Universität Berlin.

Lünenborg, Margret (1997): Journalistinnen in Europa. Eine international vergleichende Analyse zum Gendering im sozialen System Journalismus. Westdeutscher Verlag: Opladen.

Lugert, Alfred C. (1974): Auslandskorrespondenten im internationalen Kommunikationssystem. Eine Kommunikator-Studie. Pullach bei München: Verlag Dokumentation.

Maletzke, Gerhard (1966): Interkulturelle Kommunikation und Publizistikwissenschaft. In: Publizistik 11 (3-4): 318-331.

Marten, Eckhard (1987): Zwischen Skepsis und Bewunderung. Zum Tätigkeitsprofil, Selbstverständnis und Deutschlandbild amerikanischer Auslandskorrespondenten in der Bundesrepublik Deutschland. In: Publizistik 32(1): 23-33. 
Marx, Theodor (1982): Begriff und Rechtsstatus des Auslandskorrespondenten. In: Ingrid Dickhut, Olaf Jubin \& Oskar Stodiek (Hrsg.): Publizistik als Berufung. Wirken und Werk der publizistischen Persönlichkeit Heinz-Dietrich Fischer. Eine Anthologie zum 60. Geburtstag. Bochum: Brockmeyer. 203-216.

Maxwell, J. W. (1956): US Correspondents Abroad: A Study of Backgrounds. In: Journalism Quarterly 33 (3): 346-348.

Moskau, Joachim (1974): Vom Dramatiker zum Stenographen. Das Berufsbild des Auslandskorrespondenten hat sich gewandelt. In: medium 4 (4): 6-9.

Nafroth, Katja (2002): Zur Konstruktion von Nationenbildern in der Auslandsberichterstattung: Das Japanbild der deutschen Medien im Wandel. Münster: LIT.

Neudeck, Rupert (Hrsg.) (1985): Immer auf Achse. Auslandskorrespondenten berichten. Bergisch Gladbach: Bastei Lübbe.

Pedelty, Mark (1995): War Stories: The Culture of Foreign Correspondents. New York: Routledge.

Piel, Alexandra (1999): Niederländische Korrespondenten in Deutschland. Tätigkeitsprofile und Selbstverständnis. Hagen: ISL-Verlag.

Richter, Simone (1999): Journalisten zwischen den Fronten. Kriegsberichterstattung am Beispiel Jugoslawien. Opladen/Wiesbaden: Westdeutscher Verlag.

Robinson, Piers (2002): The CNN Effect: The Myth of News, Foreign Policy and Intervention. London \& New York: Routledge.

Rühl, Manfred (1989): Organisatorischer Journalismus. Tendenzen der Redaktionsforschung. In: Max Kaase \& Winfried Schulz (Hrsg.): Massenkommunikation. Theorien, Methoden, Befunde. Opladen: Westdeutscher Verlag. 253-269.

Sange, Ralf (1989): Japanische Auslandskorrespondenten in der Bundesrepublik Deutschland. Qualifikationsmerkmale, Arbeitsbedingungen und Berufseinstellungen. In: Publizistik 34 (12): 62-77.

Schmidt, Dagmar \& Jürgen Wilke (1998): Die Darstellung des Auslands in den deutschen Medien. Ergebnisse einer Inhaltsanalyse 1995. In: Siegfried Quandt \& Wolfgang Gast (Hrsg.): Deutschland im Dialog der Kulturen. Medien, Images, Verständigung. Konstanz: UVK-Medien. 167181.

Schönbach, Klaus, Dieter Stürzebecher \& Beate Schneider (1994): Oberlehrer und Missionare? Das Selbstverständnis deutscher Journalisten. In: Friedhelm Neidhardt (Hrsg.): Öffentlichkeit, Öffentliche Meinung und soziale Bewegungen. Opladen: Westdeutscher Verlag. 139-161.

Scholl, Armin \& Siegfried Weischenberg (1998): Journalismus in der Gesellschaft. Theorie, Methodologie und Empirie. Opladen: Westdeutscher Verlag.

Schraeder, Dirk (2002): Teamfähigkeit und Fremdsprachen: Der Alltag des politischen Reisekorrespondenten. In: Journalistik Journal 5 (1): 21.

Schwanebeck, Axel (2003): Die Welt im Wohnzimmer. Was leisten Auslandsberichte im deutschen Fernsehen. In: Claudia Cippitelli \& Axel Schwanebeck (Hrsg.): Nur Krisen, Kriege, Katastrophen. Auslandsberichterstattung im deutschen Fernsehen. München: Fischer. 13-31.

Siemes, Annette (2000): Auslandskorrespondenten in Polen. Nachbarschaftsvermittler zwischen Rollenverständnis und Arbeitsrealität. Bochum: Bochumer Universitäts-Verlag.

Tern, Jürgen (1982): Auslandskorrespondenten zwischen Status und Rolle. In: Ingrid Dickhut, Olaf Jubin \& Oskar Stodiek (Hrsg.): Publizistik als Berufung. Wirken und Werk der publizistischen Persönlichkeit Heinz-Dietrich Fischer. Eine Anthologie zum 60. Geburtstag. Bochum: Brockmeyer. 217-226.

Wagner, Martin (2001): Auslandskorrespondent/in für Presse, Radio, Fernsehen und Nachrichtenagenturen. München: List.

Weaver, David H., Beam, Randal A., Brownlee, Bonnie J., Voakes, Paul S. and Wilhoit, G. Cleveland (2006): The American Journalist in the 21st Century. Mahwah, NJ: Erlbaum.

Weischenberg, Siegfried (1990): Das „Prinzip Echternach“. Zur Einführung in das Thema „Journalismus und Kompetenz“. In: Siegfried Weischenberg (Hrsg.): Journalismus und Kompetenz. Qualifizierung und Rekrutierung für Medienberufe. Opladen: Westdeutscher Verlag. 11-41. 
Weischenberg, Siegfried, Martin Löffelholz \& Armin Scholl (1993): Journalismus in Deutschland. Design und erste Befunde der Kommunikatorstudie. In: Media Perspektiven (1): 21-33.

Weischenberg, Siegfried, Martin Löffelholz \& Armin Scholl (1994): Merkmale und Einstellungen von Journalisten. „Journalismus in Deutschland“ II. In: Media Perspektiven (4): 154-167.

Weischenberg, Siegfried, Armin Scholl \& Maja Malik (2005): Journalismus in Deutschland. Arbeitspapier. Universität Hamburg.

Wilhelm, J. (1963): The Re-Appearing Foreign Correspondent: A World Survey. In: Journalism Quarterly 40 (1): 147-168.

Willnat, Lars \& David Weaver (2003): Through Their Eyes: The Work of Foreign Correspondents in the United States. In: Journalism 4 (4): 403-422.

Wolfsfeld, Gadi (1997): Promoting Peace through the News Media: Some Initial Lessons from the Oslo Peace Process. In: International Journal of Press/Politics (2) 4, 52-70.

Wu, H. Denis \& John Maxwell Hamilton (2004): US Foreign Correspondents: Changes and Continuity at the Turn of the Century. In: Gazette 66 (6): 517-532. 